\title{
DESCENT OF PROJECTIVITY FOR LOCALLY FREE MODULES
}

\author{
ROGER WIEGAND ${ }^{1}$
}

\begin{abstract}
Let $R \rightarrow \hat{R}$ be the natural homomorphism from the commutative ring $R$ into its associated von Neumann regular ring, and let $M$ be a locally free $R$-module such that $\hat{R} \otimes M$ is a projective $\hat{R}$-module. We show that if $M$ is either countably generated or locally finitely generated, then $M$ is projective, and we deduce that the trace of any projective ideal is projective. These results are a consequence of a more general theorem on the descent of the Mittag-Leffler condition. The "locally free" hypothesis may be weakened to "flat" if and only if $R$ is locally perfect.
\end{abstract}

1. Introduction. Let $\phi: R \rightarrow R^{\prime}$ be a ring homomorphism and $M$ an $R$-module such that $R^{\prime} \otimes M$ is projective (as an $R^{\prime}$-module). The general problem of "descent of projectivity" is to find conditions on $M$ and $\phi$ that force $M$ to be projective. The most definitive results have traditionally required $M$ to be finitely generated and flat. Recently, however, Raynaud and Gruson have used a technique of "approximation by finitely generated modules" to obtain some remarkable theorems on descent of projectivity for nonfinitely generated modules [RG].

In the present paper we use these techniques to study the natural homomorphism $\phi: R \rightarrow \hat{R}$, where $R$ is a commutative ring and $\hat{R}$ is its associated von Neumann regular ring [O]. It was shown in [W1] that if $M$ is finitely generated and flat, and $\hat{R} \otimes M$ is projective, then $M$ is projective. Unfortunately, the hypothesis of finite generation can be dropped only for rings that are locally perfect. It seems profitable to replace flatness by the stronger assumption that $M$ be locally free. In this case, finiteness of $M$ may be replaced by either "countably generated" or "locally finitely generated". These results are easy consequences of our main theorem on the descent of the Mittag-Leffler condition.

Received by the editors December 13, 1972 and, in revised form, February 15, 1973.

AMS (MOS) subject classifications (1970). Primary 13C05, 13C10; Secondary 16A30.

Key words and phrases. Projective module, locally free module, Mittag-Leffler module, von Neumann regular ring, locally perfect ring.

1 Part of this research was supported by a grant from the National Science Foundation (GP-33548) while the author was a visitor at the University of Colorado.

(c) American Mathematical Society 1973 
All rings considered are commutative with identity. An $R$-module $M$ has a property "locally" if $M_{P}$ has that property as an $R_{P}$-module for each maximal ideal $P$. If $R \rightarrow R^{\prime}$ is a ring homomorphism, the statement “ $R$ ' $\otimes M$ has property $x$ " means " $R^{\prime} \otimes_{R} M$ has property $x$ as an $R^{\prime}$ module". If $s$ is an element of a ring $R$, the ring of fractions $\left\{\left(r / s^{n}\right)\right\}$ is denoted by $R_{s}$.

2. The main theorem. We refer the reader to the paper by Raynaud and Gruson [RG] for a thorough treatment of Mittag-Leffler modules. For convenience, we recall the pertinent definitions and results.

Let $f: A \rightarrow B$ and $g: A \rightarrow C$ be homomorphisms of $R$-modules. Then $g$ is said to dominate $f$ provided $\operatorname{Ker}\left(1_{X} \otimes f\right) \subseteq \operatorname{Ker}\left(1_{X} \otimes g\right)$ for every $R$-module $X$. We remark that if $g$ factors through $f$ then $g$ dominates $f$, and that domination is a transitive relation on maps emanating from $A$. Suppose $F$ is finitely presented and $u: F \rightarrow M$. A map $v: F \rightarrow G$ is called a stabilizer for $u$ provided $G$ is finitely presented and $u$ and $v$ dominate each other. An $R$-module $M$ is Mittag-Leffler if every map from every finitely presented module into $M$ admits a stabilizer. Examples of MittagLeffler modules include all pure-projective modules and their pure submodules. We shall need the following characterization of Mittag-Leffler modules, which is easily deduced from the proof of [RG, II, Proposition 2.1.4] and the remarks following the definition of "dominate" given above:

LEMMA 2.1. Let $\left(F_{i}, u_{j i}\right)$ be a direct system of finitely presented modules with direct limit $\left(M, u_{i}\right)$.

(1) If some $u_{i}: F_{i} \rightarrow M$ admits a stabilizer, then there is an index $j_{0} \geqq i$ such that $u_{j i}$ stabilizes $u_{i}$ whenever $j \geqq j_{0}$.

(2) If every $u_{i}$ admits a stabilizer then $M$ is Mittag-Leffler.

Every ring $R$ admits a homomorphism $\phi: R \rightarrow \hat{R}$ characterized by the following universal property: (1) $\hat{R}$ is (von Neumann) regular, and (2) every homomorphism from $R$ into a regular ring factors uniquely through $\phi$. (This was observed independently by J.-P. Olivier [O] and $\mathbf{M}$. Hochster $[\mathbf{H}]$.) The induced map ${ }^{a} \phi: \operatorname{spec}(\hat{R}) \rightarrow \operatorname{spec}(R)$ is a bijection. For each $P \in \operatorname{spec}(R), \phi$ induces an isomorphism between the fields $R_{P} / P R_{P}$ and $\hat{R}_{P}$, where $\hat{P}$ is the unique prime (=maximal) ideal of $\hat{R}$ such that ${ }^{a} \phi(\hat{P})=P$. If $\operatorname{spec}(R)$ is retopologized so as to make ${ }^{a} \phi$ a homeomorphism, the result is the (stronger) patch topology [H]. Proofs of these assertions may be found in [W2]. We now come to the main theorem.

Theorem 2.2. Let $M$ be a flat R-module. Then $M$ is Mittag-Leffler if and only if $M$ is locally Mittag-Leffler and $\hat{R} \otimes M$ is Mittag-Leffler. 
Proof. Write $M$ as the direct limit of the direct system $\left(F_{i}, u_{j i}\right)$, with each $F_{i}$ finitely presented [L, Appendice]. The "only if" implication is a direct consequence of Lemma 2.1. To prove the converse, fix an index $i$. We need an index $j \geqq i$ such that $u_{j i}$ stabilizes $u_{i}: F_{i} \rightarrow M$.

Let $P$ be a prime ideal, fixed for the moment. Since $M$ is locally MittagLeffler, Lemma 2.1 implies that $M_{P}$ is a Mittag-Leffler $R_{P}$-module. (It is necessary to work with all primes-not just the maximal ones-since we will eventually need a topological condition that depends on the existence of generic points.) By [RG, II, 2.2.1], $\operatorname{Im}\left(\left(u_{i}\right)_{P}\right)$ is contained in a countably generated, pure-projective, pure $R_{P}$-submodule $F$ of $M_{P}$. Since $F$ is flat, it is projective, and hence free. Then $\operatorname{Im}\left(\left(u_{i}\right)_{P}\right)$ is contained in a finitely generated free summand of $F$. It follows that there exist a free $R$-module $L$ of finite rank and a map $w: L \rightarrow M$ such that $w_{P}: L_{P} \rightarrow M_{P}$ is a pure monomorphism and $\operatorname{Im}\left(\left(u_{i}\right)_{P}\right) \subseteq \operatorname{Im}\left(w_{P}\right)$. (At this point we are reproducing part of the proof of [RG, II, 2.5.6], since that result appears to have a misprint in its statement as well as a minor obscurity in its proof.)

Let $U=\left\{Q \in \operatorname{spec}(R) \mid \operatorname{Im}\left(\left(u_{i}\right)_{Q}\right) \subseteq \operatorname{Im}\left(w_{Q}\right)\right\}$ and $V=\left\{Q \in \operatorname{spec}(R) \mid w_{Q}\right.$ is a pure monomorphism $\}$. We claim $U \cap V$ is a neighborhood of $P$. Assuming this for the time being, we can easily complete the proof as follows: Choose an element $s \in R$ such that $P \in D(s) \subseteq U \cap V$, where $D(s)$ is the set of primes not containing $P$. Identifying $\operatorname{spec}\left(R_{s}\right)$ with $D(s)$ and globalizing, we see that $w_{s}: L_{s} \rightarrow M_{s}$ is a pure monomorphism, and $\operatorname{Im}\left(\left(u_{i}\right)_{s}\right) \subseteq$ $\operatorname{Im}\left(w_{s}\right)$. Clearly, the map $\left(F_{i}\right)_{s} \rightarrow \operatorname{Im}\left(w_{s}\right)$ (induced by $\left.\left(u_{i}\right)_{s}\right)$ stabilizes $\left(u_{i}\right)_{s}$. By Lemma 2.1 there is an index $j_{0} \geqq i$ such that $\left(u_{j i}\right)_{s}$ stabilizes $\left(u_{i}\right)_{s}$ for each $j \geqq j_{0}$. By administering the same treatment to each $P \in$ $\operatorname{spec}(R)$, we obtain, by compactness, an index $j \geqq i$ such that $u_{j i}$ stabilizes $u_{i}$ locally, and hence globally.

To prove our claim, we observe that $U$ is open, since it is the complement of the support of the finitely generated module $\left(\operatorname{Im}\left(u_{i}\right)+\operatorname{Im}(w)\right) / \operatorname{Im}(w)$. The set $\operatorname{spec}(R)-V$ clearly contains the closure of each of its points. By the first corollary to Theorem 1 of $[\mathbf{H}]$, such a set is closed in the Zariski topology if and only if it is closed in the patch topology. Thus it suffices to show that $V$ is open in the patch topology.

Let $K$ be the kernel of $\hat{w}: \hat{R} \otimes L \rightarrow \hat{R} \otimes M$. Since $\hat{R}$ is regular, $\operatorname{Im}(\hat{w})$ is a (finitely generated) pure submodule of the Mittag-Leffler $\hat{R}$-module $\hat{R} \otimes M$, and hence is projective [RG, II, 2.1.6, 2.2.2]. Therefore $K$ is a finitely generated projective $\hat{R}$-module, and it follows that $\{Q \in \operatorname{spec}(R)$ $\left.K_{\hat{Q}}=0\right\}$ is open (and closed) in the patch topology. However, the next lemma shows that this set is precisely $V$, and hence completes the proof.

Lemma 2.3 [RG, I, 3.1.6]. Let $R$ be a local ring with residue field $k$. 
Let $u: F \rightarrow M$ be an R-homomorphism with $F$ free and $M$ flat. Then $u$ is a pure monomorphism if and only if $1_{k} \otimes u$ is a monomorphism.

The hypothesis that $M$ be flat cannot be deleted from Theorem 2.2. For example, let $R$ be a nonnoetherian ring such that $\operatorname{spec}(R)$ is noetherian, and $R_{P}$ is noetherian for each maximal ideal $P$. (Such a ring is constructed in [HO].) Let $M$ be a finitely generated module that is not finitely presented (and hence not Mittag-Leffler, by [RG, II, 2.2.2]). Clearly $M$ is locally Mittag-Leffler, and by [W2, Theorem 2] $\hat{R} \otimes M$ is projective (and hence Mittag-Leffler).

Let $j-\operatorname{spec}(R)$ denote the set of prime ideals of $R$ that are intersections of maximal ideals. As in [W3], let $\tilde{R}=\hat{R} / \cap\{\hat{P} \mid P \in j-\operatorname{spec}(R)\}$. If $j$ $\operatorname{spec}(R)$ is closed in the patch topology on $\operatorname{spec}(R)$, we say $R$ is $j$-closed. In this case the map $R \rightarrow \widetilde{R}$ induces a bijection between $\operatorname{spec}(\tilde{R})$ and $j$-spec $(R)$, and the proof of Theorem 2.2 can easily be modified to give the following result:

THeORem 2.4. Suppose $R$ is $j$-closed and $M$ is a flat $R$-module. Then $M$ is Mittag-Leffler if and only if $M$ is locally Mittag-Leffler and $\tilde{R} \otimes M$ is Mittag-Leffler.

3. Applications to the descent of projectivity. According to [RG, II, 2.2.2], a countably generated flat module is Mittag-Leffler if and only if it is projective. The following is therefore an immediate consequence of Theorem 2.2:

Proposition 3.1. A countably generated $R$-module $M$ is projective if and only if $M$ is locally free and $\hat{R} \otimes M$ is projective.

It is unknown to the author whether the countability hypothesis can be dropped, but it can be replaced by local finiteness.

Proposition 3.2. Let $M$ be a locally finitely generated $R$-module. Then $M$ is projective if and only if $M$ is locally free and $\hat{R} \otimes M$ is projective.

Proof. Write $\hat{R} \otimes M=\bigoplus \sum Q_{i}(i \in I)$, where each $Q_{i}$ is a countably generated $\hat{R}$-module, and let $x$ be an element of $M$. By Theorem 2.2, $M$ is Mittag-Leffler, and the proof of [RG, II, 3.1.3] provides a countably generated, pure submodule $M^{\prime}$ of $M$ such that $x \in M^{\prime}$ and $\hat{R} \otimes M^{\prime}=$ $\bigoplus \sum Q_{j}(j \in J)$ for some $J \subseteq I$. Then $M^{\prime}$ is locally free, and hence locally finitely generated, and it follows from purity that $M / M^{\prime}$ is locally free. Since $\hat{R} \otimes\left(M / M^{\prime}\right)=\oplus \sum Q_{i}(i \in I-J)$, all our hypotheses on $M$ carry over to $M / M^{\prime}$. A transfinite induction argument, as in the proof of [RG, II, 3.1.3], completes the proof. 
COROLlaRY 3.3. Let $M$ be a projective R-module such that for each $P, M_{P}$ is free of rank 0 or 1 (for example, take $M$ to be a projective ideal of $R$ ). Then the trace ideal of $M$ is projective.

Proof. Let $T$ be the trace of $M$. Then $T_{P}$ is free of rank 0 or 1 for each $P$, and $\hat{R} \otimes T$ is the trace of $\hat{R} \otimes M$ [V]. Now $\hat{R} \otimes M$ is isomorphic to a direct sum of principal ideals $\hat{R} e_{\alpha}, e_{\alpha}=e_{\alpha}^{2} \in \hat{R}$, by [K]. Since $\hat{R}_{p} \otimes M$ has rank 0 or 1 for each $P$, it follows easily that the idempotents $e_{\alpha}$ are orthogonal, and hence $\hat{R} \otimes M$ is isomorphic to an ideal $I$ of $\hat{R}$. Let $e=e^{2} \in I$ and let $f \in \operatorname{Hom}_{\hat{R}}(I, \hat{R})$. Then $f(e)=e f(e) \in I$. Since $I$ is generated by its idempotents, it follows that $I$ is its own trace. Thus $\hat{R} \otimes T=I$, and since $I$ is projective, Proposition 3.2 implies that $T$ is projective.

If one could get rid of the countability hypothesis in Proposition 3.1, the resulting theorem and its $\widetilde{R}$-analogue would have many applications to homological dimension theory. For example, it would follow that the homological dimension of a flat module $M$ is the maximum of $\sup _{P}$ h. $\operatorname{dim}_{R_{P}} M_{P}$ and h. $\operatorname{dim}_{\hat{R}}(\hat{R} \otimes M)$. The usefulness of results like this would stem from the fact that it is comparatively easy to get bounds on the global dimensions of $\hat{R}$ and $\tilde{R}$. For example, if $j-\operatorname{spec}(R)$ is a noetherian space of dimension $d$, then $R$ is $j$-closed, and $\operatorname{gl} \operatorname{dim}(\tilde{R}) \leqq d$, by [W3, $1.1,5.3]$ and [W2, Proposition 4].

4. Flatness is not enough. For which rings $R$ can one replace "locally free" by "flat" in the hypotheses of Proposition 3.1? The class of rings in question is rather small, but provides a natural, common generalization of "regular" and "perfect".

Proposition 4.1. The following conditions on a ring $R$ are equivalent:

(i) (resp. (ii)) If $M$ is an arbitrary (resp. a countably generated) flat $R$-module, and $\hat{R} \otimes M$ is projective, then $M$ is projective.

(iii) The nilradical of $R$ is $T$-nilpotent, and $\operatorname{dim}(R)=0$ (that is, primes are maximal).

(iv) $R_{P}$ is perfect for every maximal ideal $P$.

Proof. The program is to show that (iii) $\Rightarrow$ (iv) $\Rightarrow$ (ii) and (iii) $\Rightarrow$ (i) $\Rightarrow$ (ii) $\Rightarrow$ (iii). Assume (iii) holds, let $N$ be the nilradical of $R$, and let $\left\{x_{k}\right\}$ be a sequence of elements in a maximal ideal $P$. Since $P R_{P}$ is a nilideal of $R_{P}$, there exist elements $s_{k} \in R-P$ such that $s_{k} x_{k} \in N$. Then, for some $n, s_{1} \cdots s_{n} x_{1} \cdots x_{n}=0$, and it follows that $P R_{P}$ is $T$-nilpotent. Therefore $R_{P}$ is perfect, and (iv) is verified. Since flats are projective over a perfect ring [B], (iv) implies (ii) by Proposition 3.1.

Suppose (iii) is satisfied. Then $R / N$ is regular, since its localizations are 0 -dimensional local rings with no nilpotents, that is, fields. Therefore $R / N=\hat{R}$, and since $N$ is $T$-nilpotent, it follows that (i) is satisfied, 
by [RG, II, 1.2.6, 3.1.4(1)]. Obviously, (i) implies (ii), and the proof will be complete once we check that (ii) implies (iii).

Let $\left\{x_{k}\right\}$ be a sequence of elements of $N$. We need an $n$ such that $x_{1} \cdots$ $x_{n}=0$. As in [RG, II, 1.2.6], let $M$ be the direct limit of the system $R \rightarrow^{x_{1}} R \rightarrow x^{x_{2}} R \rightarrow \cdots$. Then $M$ is a countably generated flat $R$-module, and $(R / N) \otimes M=0$. Since $\phi$ factors through the natural map $\pi: R \rightarrow R / N$, we see that $\hat{R} \otimes M=0$. By condition (ii), $M$ is projective, and it follows from [B, 2.7] that $M=0$. Hence $x_{1} \cdots x_{n}=0$ for a suitable $n$. To show that $\operatorname{dim}(R)=0$, let $s$ be an arbitrary element of $R$. Then $R_{s}$ is a countably generated, flat $R$-module, and $\hat{R} \otimes R_{s}$ is projective by [W1, Corollary 2]. By (ii) $R_{s}$ is projective, and a typical "dual basis" argument (e.g. [CE, p. 132]) shows that $R_{s}$ is finitely generated, and hence cyclic. Therefore, for some $n$, we have $R s^{n}=R s^{n+1}$. The following observation completes the proof:

Proposition 4.2. Let $R$ be any ring. Then $\operatorname{dim}(R)=0$ if and only if principal ideals are eventually idempotent (that is, for each $x \in R$ there is an integer $n$, depending on $x$, such that $\left.R x^{n}=R x^{n+1}\right)$.

Proof. If $\operatorname{dim}(R)=0$, then for each $x$ there is a $y$ such that $x-x y x$ is nilpotent. Expanding $x^{n}(1-y x)^{n}=0$, we get $x^{n} \in R x^{n+1}$. Conversely, if principal ideals of $R$ are eventually idempotent, the same holds for every homomorphic image of $R$. Clearly, then, $R / P$ is a field for each prime $P$.

An interesting consequence of Proposition 4.2 is that taking direct products can increase Krull dimension. For example, the ideal generated by 2 in the ring $\prod_{n} Z /\left(2^{n}\right)$ is not eventually idempotent.

\section{REFERENCES}

[B] H. Bass, Finitistic dimension and a homological generalization of semi-primary rings, Trans. Amer. Math. Soc. 95 (1960), 466-488. MR 28 \#1212.

[CE] H. Cartan and S. Eilenberg, Homological algebra, Princeton Univ. Press, Princeton, N.J., 1956. MR 17, 1040.

[H] M. Hochster, Prime ideal structure in commutative rings, Trans. Amer. Math. Soc. 142 (1969), 43-60. MR 40 \#4257.

[HO] W. Heinzer and J. Ohm, Locally noetherian commutative rings, Trans. Amer. Math. Soc. 158 (1971), 273-284. MR 43 \#6192.

[K] I. Kaplansky, Projective modules, Ann. of Math. (2) 68 (1958), 372-377. MR 20 \#6453.

[L] D. Lazard, Autour de la platitude, Bull. Soc. Math. France 97 (1969), 81-128. MR 40 \#7310.

[O] J.-P. Olivier, Anneaux absolument plats universels et épimorphismes d'anneaux, C.R. Acad. Sci. Paris Sér. A-B 266 (1968), A317-A318. MR 39 \#197d.

[RG] M. Raynaud and L. Gruson, Criteres de platitude et de projectivité, Invent. Math. 13 (1971), 1-89. 
[V] W. Vasconcelos, On projective modules of finite rank, Proc. Amer. Math. Soc. 22 (1969), 430-433. MR 39 \#4134.

[W1] R. Wiegand, Globalization theorems for locally finitely generated modules, Pacific J. Math. 39 (1971), 269-274.

[W2] —, Modules over universal regular rings, Pacific J. Math. 39 (1971), 807819.

[W3] - Generators of modules over commutative rings, J. Algebra (to appear).

Department of Mathematics, University of Nebraska, Lincoln, Nebraska 68508 